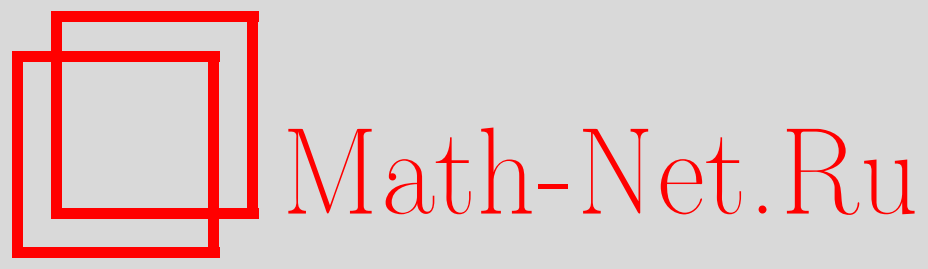

М. С. Бичегкуев, О спектре разностных и дифференциальных операторов в весовых пространствах, Функи. анализ и его прил., 2010, том 44, выпуск 1, 80-83

DOI: https://doi.org/10.4213/faa2978

Использование Общероссийского математического портала MathNet.Ru подразумевает, что вы прочитали и согласны с пользовательским соглашением

http://www . mathnet.ru/rus/agreement

Параметры загрузки:

IP : 54.166 .219 .16

26 апреля 2023 г., 12:52:42

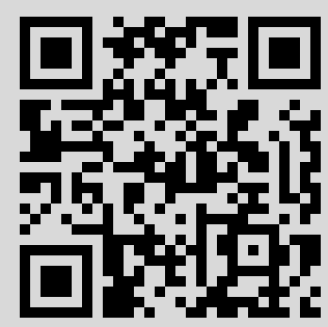


УДК 517.9

\section{О спектре разностных и дифференциальных операторов в весовых пространствах*}

(c) 2010. М. С. БичегКУЕВ

1. Пусть $X$ - комплексное банахово пространство и $L B(X)$ - банахова алгебра линейных ограниченных операторов, действующих в $X$. Рассматривается функция (вес или весовая функция) $\alpha: \mathbb{Z} \rightarrow(0, \infty)$, определенная на группе $\mathbb{Z}$ целых чисел. Символом $l_{\alpha}^{p}=l_{\alpha}^{p}(\mathbb{Z}, X)$, где $p \in[1, \infty]$, обозначим банахово пространство (двусторонних) последовательностей $x: \mathbb{Z} \rightarrow X$ векторов из $X$, суммируемых с весом $\alpha$,

$$
\|x\|=\|x\|_{p, \alpha}=\left(\sum_{n \in \mathbb{Z}}\left(\frac{\|x(n)\|}{\alpha(n)}\right)^{p}\right)^{1 / p},
$$

если $p \in[1, \infty)$, и ограниченных относительно $\alpha$,

$$
\|x\|=\|x\|_{\infty, \alpha}=\sup _{n \in \mathbb{Z}} \frac{\|x(n)\|}{\alpha(n)},
$$

если $p=\infty$. Если $\alpha \equiv 1$, то пространство $l_{\alpha}^{p}(\mathbb{Z}, X)$ обозначается через $l^{p}=$ $l^{p}(\mathbb{Z}, X)$.

Всюду считается, что весовая функция $\alpha$ удовлетворяет условию

$$
\sup _{n \in \mathbb{Z}} \frac{\alpha(n-1)}{\alpha(n)}<\infty .
$$

В заметке описан спектр оператора $\mathscr{K}: l_{\alpha}^{p}(\mathbb{Z}, X) \rightarrow l_{\alpha}^{p}(\mathbb{Z}, X)$, определенного с помощью равенств

$$
(\mathscr{K} x)(n)=A x(n-1), \quad n \in \mathbb{Z}, x \in l_{\alpha}^{p}(\mathbb{Z}, X),
$$

где $A \in L B(X)$, и получены приложения к исследованию дифференциальных операторов, действующих в весовых функциональных пространствах. Основные результаты получены с использованием величин

$$
\begin{aligned}
& æ_{\text {out }}(\alpha)=\lim _{n \rightarrow \infty}\left(\sup _{k \in \mathbb{Z}} \frac{\alpha(k)}{\alpha(k+n)}\right)^{1 / n}, \\
& æ_{\text {int }}(\alpha)=\lim _{n \rightarrow \infty}\left(\inf _{k \in \mathbb{Z}} \frac{\alpha(k)}{\alpha(k+n)}\right)^{1 / n},
\end{aligned}
$$

построенных по весу $\alpha: \mathbb{Z} \rightarrow(0, \infty)$.

Теорема 1. Спектр $\sigma(\mathscr{K})$ оператора $\mathscr{K}$ представим в виде $\sigma(\mathscr{K})=\mathbb{T} \sigma(A)\left[æ_{\text {int }}(\alpha), æ_{\text {out }}(\alpha)\right]=\left\{\gamma \lambda s: \gamma \in \mathbb{T}, \lambda \in \sigma(A), s \in\left[æ_{\text {int }}(\alpha), æ_{\text {out }(\alpha)}\right]\right\}$, где $\mathbb{T}=\{\gamma \in \mathbb{C}:|\gamma|=1\}$ - единичная окружность. 
При доказательстве теоремы 1 используется тот факт, что рассмотрение спектра оператора $\mathscr{K}$ в весовом пространстве $l_{\alpha}^{p}(\mathbb{Z}, X)$ эквивалентно рассмотрению спектра оператора $\mathscr{K}_{\Phi}=A \Phi V$ в пространстве $l^{p}(\mathbb{Z}, X)$ без веса, где $(\Phi x)(n)=$ $\Phi(n) x(n), \Phi(n)=\alpha^{-1}(n) \alpha(n-1)$ и $(V x)(n)=x(n-1)$. Спектр оператора $\mathscr{K}_{\Phi}$ инвариантен относительно вращений. Таким образом, его описание сводится к описанию компонент связности - колец. Ключевым шагом доказательства теоремы 1, позволяющим связать эти кольца, является следующая

Лемма. Если $\sigma\left(\mathscr{K}_{\Phi}\right) \cap \mathbb{T}=\varnothing$, то проектор Рисса $\mathscr{P}_{\text {int }}$, отвечающий части спектра оператора $\mathscr{K}_{\Phi}$, лежсащей внутри $\mathbb{T}$, имеет вид $\left(\mathscr{P}_{\text {int }} x\right)(n)=P_{\text {int }} x(n)$, $n \in \mathbb{Z}, x \in l^{p}(\mathbb{Z}, X)$, әде $P_{\mathrm{int}}: X \rightarrow X$ - проектор, коммутируюший с оператором $A$.

Замечание. Операторы $\mathscr{K}$ и $\mathscr{K}_{\Phi}$ являются операторами типа взвешенного сдвига. Исследованию спектра таких операторов посвящена обширная литература (см., например, [1] и цитируемые там источники). В [1], в частности, полностью описан спектр операторов взвешенного сдвига с весами из коммутативных банаховых алгебр в терминах индуцируемых динамических систем и соответствующих инвариантных мер (теоремы 4.4, 7.2, 7.7, 13.10, 17.1, 17.3). Там же доказано, что спектр упомянутого выше оператора $\Phi V$ является кольцом с центром в нуле и радиусами æ⿸ в терминах спектров операторов утверждение теоремы 1 выглядит следующим образом: $\sigma(\mathscr{K})=\sigma\left(\mathscr{K}_{\Phi}\right)=\sigma(A) \sigma(\Phi V)$.

Отметим также, что в гильбертовой ситуации теорема 1 непосредственно вытекает из результатов монографии [1]. Действительно, в пространстве $l^{2}(\mathbb{Z}, X)$, где $X$ - гильбертово пространство, рассмотрение спектра оператора $\mathscr{K}_{\Phi}=$ $A \Phi V$ эквивалентно рассмотрению спектра оператора $A \otimes \Phi V$. Как известно, спектр тензорного произведения ограниченных операторов равен произведению их спектров, т. е. $\sigma\left(\mathscr{K}_{\Phi}\right)=\sigma(A \otimes \Phi V)=\sigma(A) \sigma(\Phi V)$.

В связи с приведенной леммой подчеркнем, что при исследовании общих операторов взвешенного сдвига принципиальным является описание соответствующих спектральных проекторов Рисса. В [1] такое описание приведено в теоремах 7.2, 7.7 и лемме 19.1 .

2. Теперь рассмотрим приложения сформулированного результата к исследованию дифференциальных операторов в весовых функциональных пространствах. Операторы будут определяться с помощью семейства эволюционных операторов.

Под семейством эволюиионных операторов на банаховом пространстве $X$ понимается сильно непрерывная операторнозначная функция $\mathscr{U}: \Delta=\{(t, s) \in$ $\left.\mathbb{R}^{2}: s \leqslant t\right\} \rightarrow L B(X)$ со следующими свойствами: 1$) \mathscr{U}(t, t)=I, t \in \mathbb{R}$; 2) $\mathscr{U}(t, s) \mathscr{U}(s, \tau)=\mathscr{U}(t, \tau)$ для всех $\tau \leqslant s \leqslant t$ из $\mathbb{R} ; 3)$ существуют постоянные $M \geqslant 1, \gamma \in \mathbb{R}$, такие, что $\|\mathscr{U}(t, s)\| \leqslant M \exp \gamma(t-s)$ для всех $(t, s) \in \Delta$. Отметим, что условие 3$)$ эквивалентно условию $\sup _{(t, s) \in \Delta, 0 \leqslant t-s \leqslant 1}\|\mathscr{U}(t, s)\|<\infty$.

Функцию $\widetilde{\alpha}: \mathbb{R} \rightarrow(0, \infty)$ назовем допустимым весом, если (скалярное) семейство

$$
\mathscr{U}_{\widetilde{\alpha}}: \Delta \rightarrow L B(\mathbb{R}) \approx \mathbb{R}, \quad \mathscr{U}_{\widetilde{\alpha}}(t, s)=\frac{\widetilde{\alpha}(s)}{\widetilde{\alpha}(t)}, \quad(t, s) \in \Delta,
$$


является семейством эволюционных операторов. Ясно, что функция $\widetilde{\alpha}$ непрерывна.

Для допустимого веса $\widetilde{\alpha}: \mathbb{R} \rightarrow(0, \infty)$ символом $L_{\widetilde{\alpha}}^{p}(\mathbb{R}, X)$ обозначим банахово пространство измеримых (по Бохнеру) функций $x: \mathbb{R} \rightarrow \mathbb{X}$, для которых конечна величина

$$
\|x\|=\|x\|_{\widetilde{\alpha}, p}=\left(\int_{\mathbb{R}}\left(\frac{\|x(\tau)\|}{\alpha(\tau)}\right)^{p} d \tau\right)^{1 / p}, \quad \text { если } p \in[1, \infty),
$$

и величина

$$
\|x\|=\|x\|_{\widetilde{\alpha}, \infty}=\underset{t \in \mathbb{R}}{\operatorname{vrai} \sup } \frac{\|x(t)\|}{\widetilde{\alpha}(t)}, \quad \text { если } p=\infty .
$$

Через $C_{b, \widetilde{\alpha}}(\mathbb{R}, X)$ обозначим банахово пространство непрерывных функций $x: \mathbb{R}$ $\rightarrow X$, для которых конечна величина из формулы $(3)$. Наконец, через $S_{\widetilde{\alpha}}^{p}(\mathbb{R}, X)$ обозначим банахово пространство измеримых функций $x: \mathbb{R} \rightarrow X$, для которых

$$
\|x\|=\|x\|_{S_{\widetilde{\alpha}}^{p}}=\sup _{t \in \mathbb{R}}\left(\int_{t}^{t+1}\left(\frac{\|x(\tau)\|}{\widetilde{\alpha}(\tau)}\right)^{p} d \tau\right)^{1 / p}<\infty, \quad p \in[1, \infty) .
$$

Далее символом $\mathscr{F} \widetilde{\alpha}(\mathbb{R}, X)$ будет обозначаться одно из перечисленных функциональных пространств. Если $\alpha \equiv 1$, то соответствующие пространства будут обозначаться через $L^{p}(\mathbb{R}, X), C_{b}(\mathbb{R}, X), S^{p}(\mathbb{R}, X)$, а вместо $\mathscr{F} \widetilde{\alpha}(\mathbb{R}, X)$ будет использоваться обозначение $\mathscr{F}(\mathbb{R}, X)$.

Пусть $\mathscr{U}: \Delta \rightarrow L B(X)$ - семейство эволюционных операторов. Определим оператор $\mathscr{L}_{\mathscr{U}}: D\left(\mathscr{L}_{\mathscr{U}}\right) \subset \mathscr{F}_{\widetilde{\alpha}}(\mathbb{R}, X) \rightarrow \mathscr{F}_{\widetilde{\alpha}}(\mathbb{R}, X)$ следующим образом. Пару функций $(x, f) \in \mathscr{F}_{\widetilde{\alpha}}(\mathbb{R}, X) \times \mathscr{F}_{\widetilde{\alpha}}(\mathbb{R}, X)$ отнесем к графику оператора $\mathscr{L}_{\mathscr{U}}$, если для всех $(t, s) \in \Delta$ имеют место равенства

$$
x(t)=\mathscr{U}(t, s) x(s)-\int_{s}^{t} \mathscr{U}(t, \tau) f(\tau) d \tau, \quad s \leqslant t .
$$

Таким образом, $x \in D\left(\mathscr{L}_{\mathscr{U}}\right)$ и $\mathscr{L}_{\mathscr{U}} x=f$. Это определение корректно, т. е. функция $f$ единственна для данной функции $x \in \mathscr{F} \widetilde{\alpha}$. Для невесовых пространств такое определение использовалось в работах [2]-[6]. Результаты этих работ существенно используются здесь для исследования дифференциальных операторов в весовых пространствах.

Пусть $T: \mathbb{R}_{+}=[0, \infty) \rightarrow L B(X)$ - сильно непрерывная полугруппа операторов класса $C_{0}$ и $A$ - ее инфинитезимальный оператор. Тогда семейство $\mathscr{U}(t, s)=T(t-s),(t, s) \in \Delta$, является семейством эволюционных операторов. Определяемый этим семейством оператор $\mathscr{L}_{\mathscr{U}}: D\left(\mathscr{L}_{\mathscr{U}}\right) \subset \mathscr{F}_{\widetilde{\alpha}}(\mathbb{R}, X) \rightarrow$ $\mathscr{F}_{\widetilde{\alpha}}(\mathbb{R}, X)$ обозначим через $\mathscr{L}, \mathscr{L}=-d / d t+A$.

Далее, через $\alpha$ обозначим сужение веса $\widetilde{\alpha}: \mathbb{R} \rightarrow(0, \infty)$ на $\mathbb{Z}$ и наряду с оператором $\mathscr{L}: D(\mathscr{L}) \subset \mathscr{F}(\mathbb{R}, X) \rightarrow \mathscr{F}(\mathbb{R}, X)$ рассмотрим разностный оператор $\mathscr{K}: \mathscr{F}_{\alpha}(\mathbb{Z}, X) \rightarrow \mathscr{F}_{\alpha}(\mathbb{Z}, X),(\mathscr{K} x)(n)=T(1) x(n-1), n \in \mathbb{Z}, x \in \mathscr{F}_{\alpha}(\mathbb{Z}, X)$. Здесь банахово пространство $\mathscr{F}_{\alpha}(\mathbb{Z}, X)$ совпадает с пространством $l_{\alpha}^{p}(\mathbb{Z}, X)$, если $\mathscr{F}_{\alpha}(\mathbb{R}, X)=L_{\widetilde{\alpha}}^{p}(\mathbb{R}, X), p \in[1, \infty)$, и $\mathscr{F}_{\alpha}(\mathbb{Z}, X)=l_{\alpha}^{\infty}(\mathbb{Z}, X)$, если $\mathscr{F}_{\alpha}(\mathbb{R}, X)=$ $S_{\widetilde{\alpha}}^{p}(\mathbb{R}, X), 1 \leqslant p \leqslant \infty$, или $\mathscr{F}_{\alpha}(\mathbb{R}, X)=C_{b, \widetilde{\alpha}}(\mathbb{R}, X)$. В этом случае пространство $\mathscr{F}_{\alpha}(\mathbb{Z}, X)$ называется ассоииированным с пространством $\mathscr{F} \widetilde{\alpha}(\mathbb{R}, X)$.

Из приведенной выше теоремы 1 и теоремы 2 (об отображении спектра) из [4] вытекает следующая 
Теорема 2. Если $æ_{\text {int }}(\alpha)>0$, mo

$$
\sigma(\mathscr{L})=\left\{\lambda \in \mathbb{C}: \exp \lambda \in \mathbb{T} \sigma(T(1))\left[æ_{\text {int }}(\alpha), æ_{\text {out }}(\alpha)\right]\right\} .
$$

Eсли $æ_{\text {int }}(\alpha)=0, æ_{\text {out }}(\alpha)>0$, то справедливо представление $\sigma(\mathscr{L})=\{\lambda \in \mathbb{C}:$ $\left.\operatorname{Re} \lambda \leqslant \chi_{g}(T) \ln æ_{\text {out }}(\alpha)\right\}$, где $\chi_{g}(T)=\lim _{t \rightarrow \infty}(\ln \|T(t)\|) / t-$ показатель роста полугруппы операторов T. Cпектр оператора $\mathscr{L}$ nуст при $æ_{\text {out }}(\alpha)=0$.

Следствие 1. Оператор $\mathscr{L}: D(\mathscr{L}) \subset \mathscr{F}_{\widetilde{\alpha}}(\mathbb{R}, X) \rightarrow \mathscr{F}_{\widetilde{\alpha}}(\mathbb{R}, X)$ непрерывно обратим тогда и только тогда, когда непрерывно обратим разностный оператор $\mathscr{D}=I-\mathscr{K}: \mathscr{F}_{\alpha}(\mathbb{Z}, X) \rightarrow \mathscr{F}_{\alpha}(\mathbb{Z}, X)$, действующий в ассочиированном $c$ $\mathscr{F}_{\alpha}(\mathbb{R}, X)$ пространстве $\mathscr{F}_{\alpha}(\mathbb{Z}, X)$.

Следствие 2. Спектр оператора дифферениирования $\mathscr{L}_{0}=-d / d t: D\left(\mathscr{L}_{0}\right) \subset$ $\mathscr{F}_{\widetilde{\alpha}}(\mathbb{R}, X) \rightarrow \mathscr{F}_{\widetilde{\alpha}}(\mathbb{R}, X)$, где $D\left(\mathscr{L}_{0}\right)=\left\{x \in \mathscr{F}_{\widetilde{\alpha}}(\mathbb{R}, X): x-\right.$ абсолютно непрерьвная функиия $\left.c \dot{x} \in \mathscr{F}_{\widetilde{\alpha}}(\mathbb{R}, X)\right\}$ совпадает с множеством $\{\lambda \in \mathbb{C}: \exp \lambda \in$ $\left.\mathbb{T}\left[æ_{\text {int }}(\alpha), æ_{\text {out }}(\alpha)\right]\right\}$, если $\mathfrak{i n t}_{\text {int }}(\alpha)>0$, и с множеством $\sigma\left(\mathscr{L}_{0}\right)=\{\lambda \in \mathbb{C}: \operatorname{Re} \lambda \leqslant$ $\left.\ln æ_{\text {out }}(\alpha)\right\}$, если $\varlimsup_{\text {int }}(\alpha)=0, æ_{\text {out }}(\alpha)>0$. Спектр оператора $\mathscr{L}_{0}$ пуст, если $æ_{\text {out }}(\alpha)=0$.

Отметим, что теорема 2 может быть обобщена на дифференциальные включения [7].

Автор искренне благодарен рецензенту за ряд полезных замечаний.

\section{ЛИТЕРАТУРА}

[1] A. Antonevich, A. Lebedev, Functional Differential Equations: I. $C^{*}$-theory, Longman Scientific \& Technical, Harlow, 1994. [2] В. В. Жиков, Изв. АН СССР, сер. матем., 40:6 (1976), 1380-1408. [3] В. В. Жиков, Б. М. Левитан, Почти периодические функции и дифференииальные уравнения, Изд-во МГУ, М., 1978. [4] А. Г. Баскаков, Функц. анализ и его прил., 30:3 (1996), 1-11. [5] А. Г. Баскаков, Известия РАН, cep. матем., 73:2 (2009), 3-68. [6] C. Chicone, Y. Latushkin, Evolution Semigroups in Dynamical Systems and Differential Equations, Amer. Math. Soc., Providence, RI, 1999. [7] М. С. Бичегкуев, Матем. заметки, 79:4 (2006), 483-487.

Северо-Осетинский государственный университет им. К. Л. Хетагурова

e-mail: bichegkuev@yandex.ru

Поступило в редакцию 5 июня 2008 г.

\section{УДК 517.988 .63}

\section{Многомерная версия принципа обобщенного сжатия М. А. Красносельского}

(C) 2010. А. И. ПЕРов

1. Поводом для написания настоящей работы послужила теорема из $[1$, с. 153-154], которую мы ниже приведем в несколько измененных обозначениях (теорема 1).

Пусть $M-$ произвольное множество и $F-$ некоторое однозначное отображение этого множества в себя. Рассмотрим уравнение

$$
x=F x \quad(x \in M),
$$

